\title{
THE NUMBER OF GROUPS OF A GIVEN GENUS
}

BY

\author{
T. W. TUCKER ${ }^{1}$
}

\begin{abstract}
It is shown that the number of groups with a given genus greater than one is finite. The proof depends heavily on V. K. Proulx's classification of groups of genus one. The key observation is that as the number of vertices of a graph imbedded on a given surface increases, the average face size of the imbedding approaches the average face size of a toroidal imbedding.

The result appears to be related to Hurwitz's theorem bounding the order of a group of conformal automorphisms on a Riemann surface of genus $\mathbf{g}$.
\end{abstract}

1. Introduction. The genus of a group is the smallest genus of any of its Cayley graphs. The groups of genus 0 were classified by Maschke [5]. Groups of genus 1 have recently been classified by V. K. Proulx [6], [7]. (R. P. Baker [1] determined which Cayley graphs have "half regular" imbeddings in a torus.) In both cases, there are infinite classes of groups; for example, all cyclic and dihedral groups are planar, and any 2-generator abelian group where neither generator has order 2 is toroidal. Most other results in the literature concern computation of the genera of certain classes of groups (for a general reference, see [9] or [10]). The interest of this paper is graphs of a given genus.

A Cayley graph is regular. In $\S 2$, we construct an infinite number of regular graphs of degree 3 or 4 having a given genus. Thus the available evidence from genus 0 and 1 and from regular graphs would suggest that the number of groups of any given genus is infinite. The principal result of this paper is that in fact the number of groups of a given genus greater than one is finite. Indeed, we show that any group of genus $\gamma$, where $\gamma>1$, has order at most $168(\gamma-1)$.

The proof of this theorem relies heavily on Proulx's classification of toroidal groups. The important observation is that Proulx's arguments depend upon the average face size of an imbedding and that the average face size of an imbedding of a regular graph in a given surface approaches the average face size of a toroidal imbedding as the number of vertices of the graph increases.

Hurwitz's theorem [8, p. 92] states that the order of any group of conformal automorphisms of a Riemann surface of genus $\gamma>1$ is bounded by $84(\gamma-1)$. It is tempting to use Hurwitz's theorem to obtain our results. Indeed, suppose the group $G$ has genus $\gamma>1$ and $K$ is a Cayley graph for $G$ imbedded in a surface $S$ of genus $\gamma$. There is a natural action of $G$ on $K$. If the action can be extended to an orientation preserving action on the surface $S$, it can be shown that $G$ is a group of

Received by the editors September 15, 1978 and, in revised form, March 29, 1979.

AMS (MOS) subject classifications (1970). Primary 05C10; Secondary 05C25, 55A15, 30A58.

Key words and phrases. Cayley graph, genus of a group, Euler characteristic, Riemann surface.

${ }^{1}$ This research was partially supported by NSF Contract MCS 76-06570. 
conformal automorphisms of a surface of genus $\gamma$. Hence the order of $G$ would be bounded by $84(\gamma-1)$.

Unfortunately, the action of $G$ on $K$ does not extend to the imbedding surface in general. For example, the existence of such an extension implies that the face structure is highly regular: the number of faces of each size must be the same at each vertex (and in the same cyclic order). Levinson and Maskit [4] give examples of planar groups having no such regular imbeddings. Part of the problem here is generators of order two whose action on the Cayley graph in some sense must be orientation reversing, but it would be foolhardy to think this is the only trouble. Nevertheless, the results of this paper suggest a much deeper connection between graph theory and Riemann surfaces than might have been hitherto expected.

I wish to thank Marvin Tretkoff for pointing out to me the relevance of Hurwitz's theorem.

2. Regular graphs. A graph in this paper is a one-dimensional, finite simplicial complex; there are no multiple edges or self-adjacencies. The preferred direction of an edge is specified by the phrase "from vertex $u$ to vertex $v$." The degree of a vertex is the number of edges incident at that vertex. A graph is regular of degree $d$ if every vertex in the graph has degree $d$.

A graph $K$ has genus $\gamma(K)$ (respectively, characteristic $\chi(K)$ ) if it can be imbedded in a surface of genus $\gamma(K)$ (Euler characteristic $\chi(K)$ ) but no less (no more). Note that genus refers only to orientable surfaces and that the genus $\gamma$ and Euler characteristic $\chi$ of an orientable surface are related by $\gamma=1-\chi / 2$. Note also that the characteristic of a graph is not equal to the usual Euler characteristic of the graph as a 1-complex (namely, vertices minus edges).

Let $K$ be a graph imbedded in the surface $S$. The imbedding is cellular if each component of $S-K$ is homeomorphic to an open disk. All imbeddings in this paper are assumed to be cellular. Each component of $S-K$ is called a face. The size of a face is the length of the circuit in $K$ that forms the boundary of the face. The number of faces of size $n$ for an imbedding is denoted $F_{n}$ and the total number of faces is $F$. If the Euler characteristic of the surface $S$ is $\chi$, if the number of vertices of the graph $K$ is $V$, and if the number of edges of $K$ is $E$, then Euler's equation states that $V-E+F=\chi$.

The following proposition gives an inequality relating $F_{n}, V$, and $\chi$ for a regular graph imbedding and will be used continually throughout this paper.

Proposition 1. Let $K$ be a regular graph of degree $d$ with $V$ vertices imbedded in $a$ surface of Euler characteristic $\chi$. Let $n$ be any integer greater than 1. Then

$$
(n d / 2-n-d) V+n \chi \leqslant \sum_{i=1}^{n-1}(n-i) F_{i} \text {. }
$$

Proof. All summations will begin at $i=1$ but by our definition of graph, $F_{1}=F_{2}=0$. By counting in two ways the number of sides of edges, one obtains $\Sigma^{\infty} i F_{i}=2 E$, where $E$ is the number of edges in $K$. Thus $\Sigma^{n-1} i F_{i}+n\left(F-\Sigma^{n-1} F_{i}\right)$ $<2 E$. Since $d V=2 E$ and $F=E-V+\chi, F$ and $E$ can be eliminated from the inequality and (*) is obtained. 
COROLlARY. If $K$ is a regular graph of degree $d>6$ imbedded in a surface of Euler characteristic $\chi$, then $V \leqslant-6 \chi$. In particular the number of regular graphs of degree greater than 6 imbeddable in a given surface is finite.

Proof. Let $n=3$ in the inequality (*). Then $(d / 2-3) V+3 \chi \leqslant 0$ since $F_{1}=$ $F_{2}=0$. Thus $(d-6) V \leqslant-6 \chi$. The result follows since $d \geqslant 7$.

In contrast to the above corollary, there is

THEOREM 1. There are an infinite number of regular graphs of degree 3 and 4 of given characteristic $\chi$.

Proof. Choose a triangulation of a surface of Euler characteristic $\chi$. Let $K$ be the graph consisting of the vertices and edges of the triangulation. The characteristic of $K$ is $\chi$ since no other imbedding of $K$ can have more faces (every face of the imbedding of $K$ is a triangle and $K$ has no loops or multiple edges). We "blow up" the vertex $v$ by choosing a small disk neighborhood $D$ of $v$, deleting $K \cap D$ from $K$ and adding the boundary of $D$ to $K$. (See Figure 1.)

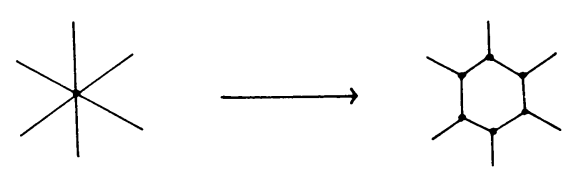

FIGURE 1. Blowing up a vertex

The graph $K^{\prime}$ obtained by blowing up each vertex of $K$ is regular of degree 3 . The graph $K$ can be recovered from $K^{\prime}$ by removing one of the added edges around each old vertex of $K$ and then contracting the other added edges. Since removing or contracting an edge can only increase the characteristic of a graph, the characteristic of $K^{\prime}$ is no larger than $\chi$. Hence it is $\chi$ as $K^{\prime}$ is already imbedded in $S$. This blowing-up process can be repeated indefinitely. Because the number of vertices is strictly increasing, an infinite number of regular graphs of degree 3 and characteristic $\chi$ are created. (See Figure 2.)

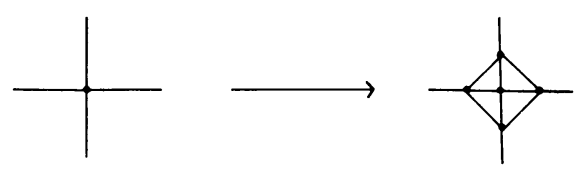

Figure 2. A new graph of degree 4 from an old one

To prove the theorem for regular graphs of degree 4 , assume first that there is a regular graph $K$ of degree 4 and given characteristic $\chi$. Choose an imbedding of $K$ in a surface of Euler characteristic $\chi$. For each vertex $v$ of $K$, choose a small disk neighborhood $D$ and add the boundary of $D$ to $K$. Call the resulting graph $K^{\prime}$. Then $K^{\prime}$ is regular of degree 4 . Since $K$ can be obtained from $K^{\prime}$ by removing edges and eliminating vertices of degree 2 , the characteristic of $K^{\prime}$ is at most $\chi$, and 
hence it is $\chi$. This process can be iterated to produce an infinite number of regular graphs of degree 4 and characteristic $\chi$.

It remains to show that there is a regular degree 4 graph $K$ of given characteristic $\chi$. Let $S$ be a surface of Euler characteristic $\chi$ and let $K$ be the edges and vertices of the first barycentric subdivision of a triangulation of $S$. Each vertex of $K$ has even degree. Now blow up each vertex of $K$ to make a regular graph $K^{\prime}$ of degree 3 and characteristic $\chi$. Around each old vertex of $K$ there is a circle of an even number of new edges of $K^{\prime}$. Double every other edge around this circle (see Figure 3 ) to obtain $K^{\prime \prime}$.

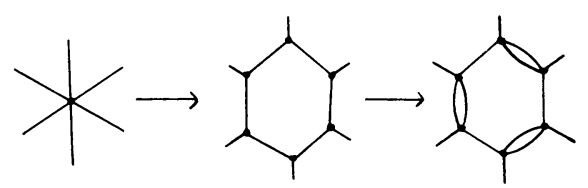

FIGURE 3. Obtaining a graph of degree 4

Then $K^{\prime \prime}$ is a regular graph of degree 4 and characteristic $\chi$. The process described in the previous paragraph can now be applied to $K^{\prime \prime}$, resulting in a graph $K^{\prime \prime \prime}$. This graph is regular of degree 4 and characteristic $\chi$ and has no multiple edges.

We do not have a method for constructing an infinite number of regular graphs of degrees 5 and 6 having a given characteristic. ${ }^{2}$ The corollary to Proposition 1 does not forbid this possibility.

3. Cayley graphs. Let $G$ be a group with identity element 1 and let $X=$ $\left\{x_{1}, \ldots x_{r}, y_{1}, \ldots y_{s}\right\}$ be a generating set for $G$ such that $x_{i}$ has order greater than two for all $i, y_{i}$ has order 2 for all $i, x_{i} \neq x_{j}^{ \pm 1}$ for $i \neq j$, and $y_{i} \neq y_{j}$ for $i \neq j$. The Cayley graph $K(G, X)$ has $G$ as vertex set, a directed edge from $w$ to $w x_{i}$ for all $w \in G$ and all $x_{i}$, and an undirected edge between $w$ and $w y_{i}$ for all $w \in G$ and all $y_{i}$. Note that $K(G, X)$ has no multiple edges or self-adjacencies because of the restrictions that $x_{i} \neq x_{j}^{ \pm 1}$ and that no generator is the identity. Also $K(G, X)$ is regular of degree $2 r+s$. To each arbitrarily directed edge of $K(G, X)$ there corresponds either an element of $X$ (if the arbitrary direction agrees with the defined direction) or the inverse of an element of $X$ (if directions disagree). To each directed path in $K(G, X)$, there corresponds a word (product) in the generators and their inverses. To each circuit there corresponds a relator in the generators (a word equal to 1).

The genus $\gamma(G)$ (characteristic $\chi(G)$ ) of the group $G$ is the smallest genus (largest characteristic) of any of its Cayley graphs. The main result of this paper, Theorem 2 , is that there are only finitely many groups of a given genus greater than one or a given characteristic less than zero. The proof is based entirely on V. K. Proulx's classification [6] of groups of genus 1. Basically, Proulx uses the average face size of

\footnotetext{
${ }^{2}$ J. L. Gross and V. K. Proulx have independently found such a method.
} 
a toroidal imbedding of a Cayley graph to obtain information about the group's presentation. When enough information is accumulated, the group falls into one of several classes of group presentations. The voltage graph construction of $\mathbf{J}$. L. Gross [2] is used to show every group from a given presentation class has an imbedding in a torus. The reason toroidal imbeddings play such an important role here is that if $A$ is the average face size of an imbedding of a regular graph of degree $d$ in a surface of Euler characteristic $\chi$, then $A=2 E / F=d /(d / 2-1+$ $\chi / V)$ so that as $V$ increases, $A$ approaches the average face size of a toroidal imbedding (where $\chi=0$ ).

Since much of the proof of our main theorem consists of manipulations of group relations, a few comments about relations are in order. A word in a set of generators of a group is a sequence of generators and inverses of generators. The length of the word is the number of terms in the sequence. A $k$-relator in the group is a word of length $k$, the product of whose terms is the identity. By abuse of language, we will usually refer to a product of generators and their inverses as a word. If $R$ is a relator then so are $R^{-1}$ and $w R w^{-1}$ for any element of the group $w$. In particular, if $x y \ldots z w$ is a relator so are $w x y \ldots z$ and any other cyclic permutation of the relator. If a proper subword of the relator $R$ is itself a relator, then $R$ can be reduced to a relator of shorter length by deleting that subword. A generating set for a group $G$ is redundant if a proper subset generates the group; otherwise a generating set is irredundant. A set of generators is redundant if and only if there is a relator in which one generator appears exactly once. Finally, $\left\langle w_{1}, \ldots, w_{t}\right\rangle$ denotes the subgroup of the group $G$ generated by the elements $w_{1}, \ldots, w_{t}$ of $G$.

THEOREM 2. Let $\chi$ be any integer less than zero and let $G$ be a group of order $|G|$ greater than $84|\chi|$. If $K(G, X)$ imbeds in a surface of Euler characteristic $\chi$ for some irredundant generating set $X$, then the genus of $G$ is 0 or 1 .

Proof. The proof will proceed by cases that depend first upon the degree of $K(G, X)$ and then upon the orders of the generators. Let $d$ be the degree of $K(G, X)$. By the corollary to Proposition 1 we may assume $d \leqslant 6$.

Since $X$ is irredundant the only 3-relators are of the form $x^{3}, x \in X$. In particular, the number of faces of size 3 meeting at any vertex is bounded by the number of generators in $X$ of order 3. Thus if $d=6$, at most 3 triangles meet at a vertex so $3 F_{3} \leqslant 3 V$ where $V$ is the number of vertices of $K(G, X)$, namely the order of $G$. By (*) of Proposition 1 with $n=4$ and $d=6,2 V+4 \chi<F_{3}$. Since $F_{3}<V$ we conclude $V \leqslant-4 \chi=4|\chi|$.

If $d=5$, at most 2 generators in $X$ have order 3, so by (*) with $n=4$ and $d=5$, $V+4 \chi<F_{3} \leqslant 2 V / 3$. We conclude that $V \leqslant 12|\chi|$.

For $d=4$, the above arguments fail to give any bound on $V$. The case $d=4$ will occupy the remainder of this section.

Case Ia. $X=\{x, y\}, x$ and $y$ have orders greater than 3. Here $F_{3}=0$. By (*) with $n=5$ and $d=4, V+5 \chi \leqslant F_{4}$. Thus $F_{4}>0$ if $V>5|x|$. Possible 4-relators are (up to renaming the generators) (1) $x y x^{-1} y^{-1}$, (2) $x y x^{-1} y$, (3) $x y x y$, (4) 
$x^{-1} y x^{-1} y$, (5) $x^{2} y^{2}$, (6) $x^{2} y^{-2}$, (7) $x^{4},(8) y^{4}$. Proulx [6, p. 97] or [7, Theorem 2] shows $K(G, X)$ has a toroidal imbedding if any of the following combinations of relators occur

(i) (1),

(ii) (2) and $\left\langle x^{2}, y\right\rangle$ has index 2 in $G$,

(iii) (5) or (6) and $\left\langle x^{-1} y, x^{2}\right\rangle$ has index 2 in $G$,

(iv) (3) and (4) and $\left\langle x^{2}, y^{2}\right\rangle$ has index 4 in $G$,

(v) (3) and (4) together with (7) and (8).

In condition (ii), Proulx shows that $\left\langle x^{2}, y\right\rangle=H$ has index at most 2 by coset enlimeration. Since

$$
1=\left(x y x^{-1} y\right)\left(y^{-1} x^{-1} y^{-1} x\right)=x y x^{-2} y^{-1} x,
$$

it follows that $x^{2} y x^{-2} y^{-1}=1$. Thus $H$ is abelian so $H=G$ gives the contradiction $y^{2}=1$. Similarly in condition (iii), $\left\langle x^{-1} y, x^{2}\right\rangle=H$ has index at most 2 in $G$ and if $H=G, G$ is abelian. For condition (iv), let $H=\left\langle x^{2}, y^{2}\right\rangle$ and let $J=\left\langle x^{2}, y\right\rangle$. Then $H \subset J$. Again, by coset enumeration $H$ has index at most 4 in $G$ and $J$ has index at most 2 in $G$. By (3) and (4),

$$
1=(x y x y)\left(y^{-1} x y^{-1} x\right)=x y x^{2} y^{-1} x \text {. }
$$

Thus $y x^{2} y^{-1} x^{2}=1$ and by the above analysis of condition (ii) (with the roles of $x$ and $y$ played by $y$ and $x^{2}$ ), $J$ has genus at most 1 and $H$ is abelian. Hence if $H=G$ or $J=G, G$ has genus 0 or 1 . If $H=J$, then $J$ is abelian and $y x^{2} y^{-1} x^{2}=1$ implies $x^{4}=1$. A similar argument with $J^{\prime}=\left\langle x, y^{2}\right\rangle$ shows $G$ has genus 0 or 1 or $y^{4}=1$. But if both $x^{4}$ and $y^{4}$ are relators, condition (v) occurs so that $G$ still has genus 0 or 1. We conclude that in condition (iv) as in conditions (iii) and (ii) the subgroup restrictions are unnecessary.

It is straightforward to verify that if 4 faces of size four meet at a vertex, one of the five conditions listed above occurs so that $G$ has genus 0 or 1 . If no vertex lies on more than three faces of size 4 , then $F_{4} \leqslant 3 V / 4$. By (*) with $n=5$ and $d=4$, $V+5 \chi \leqslant F_{4} \leqslant 3 V / 4$. We conclude that if $V>20|\chi|, G$ has genus 0 or 1 .

Case Ib. $X=\{x, y\}, x^{3}=1, y$ has order greater than 3. Here $F_{3}<V / 3$. By (*) with $n=5$ and $d=4, V+5 \chi \leqslant 2 F_{3}+F_{4} \leqslant 2 V / 3+F_{4}$. Thus $F_{4}>0$ if $V>$ $15|\chi|$. Of the 4-relators listed in Case Ia, only (3), (4), and (8) do not immediately imply $G$ has genus at most 1 .

Suppose (8) holds, that is $y^{4}=1$. If (3) or (4) is also a relator, then Proulx [6, p. 107] shows $G$ is planar of order 24 or less. Therefore, we assume $y^{4}$ is the only 4-relator. In particular, $F_{4} \leqslant V / 4$ so that by (*) with $n=6$ and $d=4,2 V+6 x \leqslant$ $3 F_{3}+2 F_{4}+F_{5} \leqslant 3(V / 3)+2 V / 4+F_{5}$. Hence if $V>12|\chi|$, then $F_{5}>0$. The only possible 5-relators are of the form $x y x^{-1} y^{ \pm 2}$ or $x y x y^{ \pm 2}$ (replace $y$ by $y^{-1}$ if necessary and recall $\left.x^{2}=x^{-1}\right)$. The first implies $x y x^{-1}=y^{ \pm 2}$ and

$$
1=y^{ \pm 4}=\left(x y x^{-1}\right)\left(x y x^{-1}\right)=x y^{2} x^{-1} \text {, }
$$

a contradiction since $y^{2} \neq 1$. The second implies

$$
1=y^{ \pm 4}=(x y x)(x y x)=x y x^{-1} y x
$$


so that $x^{-1} y x^{-1} y=1$. But as already stated, this relation implies $G$ is planar of order at most 24.

Suppose instead that the 4-relator is (3) or (4). Replacing $y$ by $y^{-1}$, we can in fact assume the 4-relator is (3), $x y x y$. Again there is no other 4-relator or else $G$ has genus 0 or 1 . Since $x y=y^{-1} x^{-1}$, the possible 5-relators discussed above reduce to $y^{1 \pm 2}$ or $y^{-1} x y^{2}$ contradicting irredundancy or $y^{3} \neq 1$. Hence $F_{5}=0$. Unfortunately, it is possible for two faces of size 4 corresponding to the relator $x y x y$ to meet at one vertex. Thus the best upper bound we have for $F_{4}$ is $2 \mathrm{~V} / 4$ and this is not small enough to use (*) to get $F_{5}>0$. We will have to consider 6-relators.

By using $x y=y^{-1} x^{-1}, y x=x^{-1} y^{-1}$, and $x^{2}=x^{-1}$, we can reduce many 6-relators to a contradictory relator or a relator that by previous analysis implies $G$ has genus 0 or 1 . The remaining 6-relators are of the forms $y^{6}, x y^{-1} x y^{ \pm 3}$, $x y^{2} x^{-1} y^{-2}, x y^{-2} x y^{-2},\left(x y^{-1}\right)^{3}$. Proulx [6, p. 107] shows $y^{6}$ with $x y x y$ gives a toroidal imbedding of $K(G, X)$. If $x y^{-1} x y^{3}=1$ then

$$
y^{ \pm 6}=\left(y^{ \pm 3}\right)^{2}=\left(x y^{-1} x\right)\left(x y^{-1} x\right)=x y^{-1} x^{-1} y^{-1} x=x^{3}=1
$$

so that again $K(G, X)$ has genus at most 1 . If $x y^{2} x^{-1} y^{-2}=1$, then $y^{-1} x^{-1} y x^{-1} y^{-2}$ $=1$ so that $x y^{-1} x y^{3}=1$, which as above implies $y^{6}=1$ again. If $x y^{-2} x y^{-2}=1$, then

$$
\begin{aligned}
y^{6} & =\left(y^{2}\right)^{3}=\left(x y^{-2} x\right)^{3}=x y^{-2}\left(x^{-1} y^{-2} x^{-1}\right) y^{-2} x \\
& =x y^{-2}(y x x y) y^{-2} x=x y^{-1} x^{-1} y^{-1} x=x^{3}=1 .
\end{aligned}
$$

Finally, if $\left(x y^{-1}\right)^{3}=1$, then

$$
\begin{aligned}
1 & =\left(x y^{-1}\right)^{3}(y x y x)=x y^{-1} x\left(y^{-1} x^{-1}\right) y x \\
& =x\left(y^{-1} x^{-1}\right) y^{2} x=x^{-1} y^{3} x .
\end{aligned}
$$

But Proulx [6, p. 107] shows that the relators $x^{3}, y^{3}$, and $x y x y$ imply $G$ is planar of order at most 12.

Thus if $F_{6}>0, G$ has genus at most 1 . Since $F_{5}=0, F_{4} \leqslant 2 V / 4$, and $F_{3} \leqslant V / 3$, by (*) with $n=7$ and $d=4,3 V+7$ and $d=4,3 V+7 \chi<4(V / 3)+3(2 V / 4)+$ $F_{6}$. Therefore $F_{6}>0$ when $V>42|\chi|$.

This concludes Case Ib.

Case Ic. $X=\{x, y\}, x^{3}=y^{3}=1$. The only 4-relator that does not immediately imply that $G$ has genus 0 or 1 is $x y x y$ but as noted above, the relators $x^{3}, y^{3}$, and $x y x y$ make $G$ a planar group of order at most 12 . Any 5-relator can be reduced since $x^{2}=x^{-1}$ and $y^{2}=y^{-1}$. Therefore we assume $F_{4}=F_{5}=0$.

By (*) with $n=7$ and $d=4,3 V+7 \chi \leqslant 4 F_{3}+F_{6} \leqslant 4(2 V / 3)+F_{6}$, so that if $V>21|x|$ then $F_{6}>0$. By renaming $x$ and $y$, by replacing a generator by its inverse, or by inverting a relator, we can reduce the list of possible 6-relators to $(x y)^{3}, x y x y x y^{-1}, x y x y x^{-1} y^{-1}, x y x y^{-1} x^{-1} y^{-1}$. Proulx [6, p. 110] shows that the first implies $K(G, X)$ has a toroidal imbedding. If $x y x y x y^{-1}=1$, then

$$
1=\left(x y x y^{-1} x y\right)\left(y^{-1} x^{-1} y^{-1} x^{-1} y x^{-1}\right)=x y x y x^{-1} y x^{-1} .
$$

Thus $y x y x^{-1} y=1$ so $x y x^{-1} y^{-1}=1$ and $G$ is abelian and toroidal. If $x y x y x^{-1} y^{-1}$ $=1$, then 


$$
\left(x y x^{-1} y^{-1} x y\right)\left(y^{-1} x^{-1} y^{-1} x^{-1} y x\right)=x y x^{-1} y x^{-1} y x
$$

so $x^{-1} y x^{-1} y x^{-1} y=1$ giving the relator $(x y)^{3}$ again when $x^{-1}$ is replaced by $x$. Finally, Proulx shows that $x y x y^{-1} x^{-1} y^{-1}=1$ implies that $G$ has order at most 24 and genus 0 or 1 .

We conclude that $G$ has genus 0 and 1 in this case whenever $V>21|\chi|$.

Case IIa. $X=\{x, y, z\}, y^{2}=z^{2}=1, x$ has order at least 4. Proulx [6, p. 113] shows any one of the following conditions implies $K(G, X)$ has a toroidal imbedding:

(i) $x y x y=x z x z=1$ and $\langle x, y z\rangle$ has index 2,

(ii) $x y x y=x z x^{-1} z=1$ and $\langle x, y z\rangle$ has index 4 ,

(iii) $x y x^{-1} y=x z x^{-1} z=1$ and $\langle x, y z\rangle$ has index 2 .

In condition (i) and (iii), $\langle x, y z\rangle=H$ has index at most 2 by coset enumeration. Also the given relators in (i) and (iii) imply $H$ is abelian so that even if $H=G, G$ has genus at most 1 . In condition (ii) let $H=\left\langle x,(y z)^{2}\right\rangle$ and $J=\langle x, y z\rangle$. By coset enumeration, $J$ has index at most 2 in $G$ and $H$ has index at most 2 in $J$. The relators of (ii) imply $H$ is abelian and $x(y z) x(y z)^{-1}=1$. Thus if $H=J$, then $x^{2}=1$ a contradiction. If $J=G$, then $x$ and $y z=w$ generate $G$ and $x w x w^{-1}=1$; the analysis of Case Ia can be applied to show $G$ has genus at most 1 . Thus if $H$ has index less than $4, G$ still has genus 0 or 1 . We conclude that the subgroup restrictions in the above conditions are unnecessary.

If $\mathbf{4}$ faces of size $\mathbf{4}$ meet at a vertex, one of the above conditions must hold. As in Case Ia, this happens when $V>20|\chi|$.

Case IIb. $X=\{x, y, z\}, x^{3}=y^{2}=z^{2}=1$. Note that any 5-relator can be reduced to a 4-relator since $x^{2}=x^{-1}$ and $y^{2}=z^{2}=1$. Consider the triangle in Figure 4 (from Proulx [6, p. 126]). One face bordering the triangle must involve $x$, $y$, and $z$ and hence has size at least 6 . If the other two faces both have size less than 6 , then one of the conditions listed in Case IIa holds and $G$ has genus 0 or 1 . Therefore we assume only one of the three faces bordering each triangle has size less than 6; in particular, two vertices of each triangle lie on at most two faces of size 4 or 5 and the other vertex lies on at most one face of size 4 or 5 .

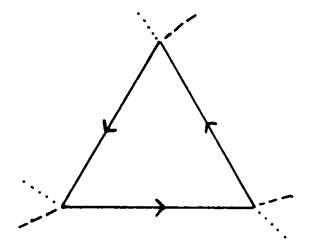

FIGURE 4. A triangle ( $x$ edges in full lines, $y$ broken, $z$ dotted)

By (*) with $n=6$ and $d=4,2 V+6 \chi \leqslant 3 F_{3}+2 F_{4}+F_{5}$. To make the right side of this inequality as large as possible, we first make $F_{3}$ as large as possible and then $F_{4}$ and $F_{5}$. Thus first let $F_{3}=V / 3$. Then by the above analysis of triangles, $4 F_{4}+5 F_{5}<2 \cdot 2 V / 3+V / 3$ so that $2 F_{4}+F_{5} \leqslant 5 V / 6$. Therefore $2 V+6 \chi<$ $3 F_{3}+2 F_{4}+F_{5} \leqslant 11 V / 6$ so that $V \leqslant 36|x|$. We conclude that if $V>36|x|, G$ has genus 0 or 1 . 
Case III. $X=\{x, y, z, w\}$, all generators have order 2. Since $F_{3}=0$, just as in Case Ia, there will be 4 faces of size 4 at one vertex if $V>20|x|$. Up to renaming the generators, we have the four 4-relators $(x y)^{2},(y z)^{2},(z w)^{2},(w x)^{2}$. Proulx [6, p. 130] shows these relators together imply $G$ has genus 0 or 1 .

4. The cases of Theorem 2 for $d=3$. In this section we complete the proof of Theorem 2 by considering the cases for $d=3$.

Case Ia. $X=\{x, y\}, x^{2} \neq 1, y^{2}=1, F_{3}=F_{4}=F_{5}=0$. Possible 6-relators are (1) $x y x^{ \pm 3} y$, (2) $x^{2} y x^{ \pm 2} y$, (3) $x y x y x^{-1} y$, (4) $(x y)^{3}$, (5) $x^{6}$. Proulx [6, p. 47] shows: (1) makes $G$ toroidal of order 16 , (2) makes $G$ have genus 0 or $1,(4)$ and (5) make $G$ have genus 0 or 1 . If (3) holds,

$$
1=\left(x y x y x^{-1} y\right)\left(y x y x^{-1} y x\right)=x^{2},
$$

a contradiction. Thus, if 3 faces of size 6 meet at a vertex, $G$ must have genus at most 1 . If no vertex lies on more than 2 hexagons $F_{6}<2 V / 6$, and by (*) with $n=7$ and $d=3, V / 2+7 \chi \leqslant F_{6} \leqslant 2 V / 6$. We conclude that if $V>42|\chi|, G$ has genus at most 1 .

Case Ib. $X=\{x, y\}, x^{2} \neq 1, y^{2}=1 F_{3}=F_{4}=0, F_{5} \neq 0$. Possible 5-relators are $x y x^{ \pm 2} y$ and $x^{5}$. If $x y x^{2} y=1$ then

$$
x^{4}=x^{2} x^{2}=\left(y x^{-1} y\right)\left(y x^{-1} y\right)=y x^{-2} y=x
$$

so that $x^{3}=1$. But then $x y x^{2} y=x y x^{-1} y$ making $G$ abelian of order 6. Similarly, $x y x^{-2} y=1$ implies $x^{3}=1$ making $G$ nonabelian of order 6 but still planar. Therefore we assume $x^{5}=1$ and $F_{5} \leqslant V / 5$.

Each of the 6-relators of Case Ia immediately implies $G$ has genus 0 or 1 except $(x y)^{3}$, but Proulx [6, p. 55] shows $(x y)^{3}=1$ with $x^{5}=1$ makes $G$ planar of order at most 60 . The only possible 7 -relators that cannot be reduced using $x^{5}=1$ are of the form $x y x^{ \pm 1} y x \pm 2 y$. If $x y x^{-1} y x^{ \pm 2} y=1$, then

$$
1=\left(x y x^{-1} y x^{ \pm 2} y\right)\left(y x^{ \pm 2} y x y x^{-1}\right)=\left(x y x^{-1} y\right) x^{ \pm 1}\left(y x y x^{-1}\right)
$$

so that $x=1$. If $x y x y x^{2} y=1$, then

$$
1=\left(x^{2} y x y x y\right)\left(y x^{-1} y x^{-2} y x^{-1}\right)=x^{2} y x^{-1} y x^{-1}
$$

so that $x y x^{-1} y=1$, and $G$ is the planar abelian group of order 10. Similarly, $x y x y x^{-2} y=1$ implies $x^{3} y x^{-3} y=1$, which again makes $G$ abelian of order 10 since $x^{3}$ and $y$ generate $G$. Thus if $F_{7}>0, G$ has genus 0 . But by (*) with $n=8$ and $d=3, V+8 \chi<3 F_{5}+F_{7} \leqslant 3 V / 5+F_{7}$. Therefore, $F_{7}>0$ and $G$ has genus at most 1 if $V>20|x|$.

Case Ic. $X=\{x, y\}, x^{2} \neq 1, y^{2}=1, F_{3}=0, F_{4} \neq 0$. The relator $x y x^{-1} y$ implies $G$ is abelian and planar, and $x y x y=1$ implies $G$ is dihedral and planar. Therefore we assume $x^{4}=1$ and $F_{4} \leqslant V / 4$. The previous analysis of 5 and 6-relators allows us to assume $F_{5}=F_{6}=0$. The only possible 7-relator is $x y x^{ \pm 1} y x^{2} y$. If $x y x y x^{2} y=$ 1 , we conclude that $G$ is abelian and planar just as in Case Ib. If $x y x^{-1} y x^{2} y=1$, then $x y x y=x^{-1} y x$ so that $(x y)^{4}=\left(x^{-1} y x\right)\left(x^{-1} y x\right)=1$. The 8-relator $(x y)^{4}$ is considered below. We therefore assume $F_{7}=0$. 
By (*) with $n=9$ and $d=3,3 V / 2+9 \chi \leqslant 5 F_{4}+F_{8} \leqslant 5 V / 4+F_{8}$ so that $F_{8}>0$ if $V>36|x|$. Possible 8-relators that cannot be reduced using $x^{4}=1$ are $(x y)^{4},(x y)^{2}\left(x^{-1} y\right)^{2},\left(x y x^{-1} y\right)^{2},(x y)^{3} x^{-1} y, x^{2} y x^{2} y x^{ \pm 1} y$. Proulx [6, p. 59] shows that any one of the first three relators implies $G$ has genus at most 1 . If $(x y)^{3} x^{-1} y=1$, then

$$
1=\left((x y)^{3} x^{-1} y\right)\left(y x y x^{-1} y x y x\right)=x y x^{2} y x
$$

so that $x^{2} y x^{2} y=1$, which as stated in Ia implies $G$ has genus 0 or 1. If $x^{2} y x^{2} y x^{ \pm 1} y$ $=1$, then

$$
1=\left(x^{ \pm 1} y x^{2} y x^{2} y\right)\left(y x^{2} y x^{2} y x^{ \pm 1}\right)=x^{ \pm 2},
$$

a contradiction. We conclude that if $V>36|x|, G$ has genus at most 1 .

Case Id. $X=\{x, y\}, x^{2} \neq 1, F_{3} \neq 0$. Of course in this case $x^{3}=1$. In particular any relator of odd length can be reduced since $x^{2}=x^{-1}$. Relators of length 4,6 , and 8 are handled by arguments in the previous cases. Proulx $[6$, p. 64] shows the possible 10-relators either lead to contradictions or imply that $G$ is planar of order at most 60 . We assume therefore that $F_{n}=0$ for $4 \leqslant n \leqslant 11$.

By (*) with $n=14$ and $d=3,4 V+14 \chi \leqslant 11 F_{3}+2 F_{12}+F_{13} \leqslant 11 V / 3+$ $2 F_{12}+F_{13}$, so that $2 F_{12}+F_{13}>0$ if $V>42|\chi|$. Since any 13-relator can be reduced to a 12-relator, there must be a 12-relator if $V>42|\chi|$. Proulx [6, p. 65] considers all possible 12-relators. Every one implies that $G$ has genus 0 or 1 except (1) $x y x y x^{-1} y x y x^{-1} y x^{-1} y$ and (2) $x y x y x^{-1} y x^{-1} y x y x^{-1} y$, but given either (1) or (2) coset enumeration for the subgroup generated by $x$ shows that the order of $G$ is at most 42.

Case IIa. $X=\{x, y, z\}, x^{2}=y^{2}=z^{2}=1, F_{3}=F_{4}=F_{5}=0$. Possible 6-relators are (up to renaming generators) (1) $(x y)^{3},(2)(x z)^{3},(3)(y z)^{3},(4)(x y z)^{2}$, (5)xzxyzy. Proulx shows [6, p. 73] that (4) by itself implies $K(G, X)$ has a toroidal imbedding. Suppose (5) holds. Let $u=x y$ and $v=y z$. Proulx shows that if $H=\left\langle u, v^{2}\right\rangle$ has index 4 in $G$, then $K(G, X)$ is toroidal. Let $J=\langle u, v\rangle$. By coset enumeration, $H$ has index 2 in $J$, and $J$ index 2 in $G$. By (5) $u v u v^{-1}=1$. Therefore, if $J=G, G$ has genus 0 or 1 by Case Ia for $d=4$. If $H=J$, then $J$ is abelian because $u v^{2} u^{-1} v^{-2}=1$. In particular,

$$
1=u v^{-1} u^{-1} v=(x y)(z y)(y x)(y z)=x y z x y z=1 ;
$$

hence (4) holds and again $G$ has genus at most 1.

Finally, suppose (1), (2), and (3) all hold. Let $u=x y$ and $v=x z$. Proulx shows [6, p. 75] that $K(G, X)$ has a toroidal imbedding if $H=\langle u, v\rangle$ has index 2 in $G$. As usual, the index of $H$ is at most 2 by coset enumeration. Also, since $u^{3}=v^{3}=$ $\left(u^{-1} v\right)^{3}=1, H$ has genus 0 or 1 by Case Ic for $d=4$. Thus even if $H=G$, still $G$ has genus 0 or 1 . If three faces of size 6 meet at a vertex either (1), (2), and (3) all hold or (4) holds or (5) holds; in any case, $G$ will have genus at most 1 . As in Case Ia for $d=3$, this will happen if $V>42|x|$.

Case IIb. $X=\{x, y, z\}, x^{2}=y^{2}=z^{2}=1, F_{4} \neq 0$. Note that $F_{3}=F_{5}=0$ automatically by the irredundancy of $X$. If more than one of the three possible 4-relators, $(x y)^{2},(x z)^{2},(y z)^{2}$, are present, Proulx [6, p. 80] shows that $G$ has genus 0. 
Therefore, we assume throughout the rest of this case that $(x y)^{2}=1,(x z)^{2} \neq 1 \neq$ $(y z)^{2}$ and $F_{4} \leqslant V / 4$.

Assume for the moment that $F_{6}=0$. It can be verified [6, p. 81] that every possible 7-relator contradicts either our assumption that $(x z)^{2} \neq 1$ and $(y z)^{2} \neq 1$ or the irredundancy of $X$. Using the fact that $x$ and $y$ commute, we reduce the list of 8-relators that do not contradict the irredundancy of $X$ to (i) $(x z)^{4}$, (ii) $(y z)^{4}$, (iii) $x z x z y z y z$, (iv) $x z y z x z y z$, (v) $x y z x y z x z$, (vi) xyzxyzyz.

If (v) holds, let $u=x y$. Then $u^{2}=1$, and $x, z, u$ generate $G$. But (v) becomes uzuzxz so that in fact $u$ and $z$ generate $G$. Since $u^{2}=z^{2}=1, G$ is a dihedral group and planar. A similar argument shows that if (vi) holds, $G$ is dihedral and planar. Proulx [6, p. 82] shows that (iv) by itself implies $K(G, X)$ is toroidal and (iii) implies $G$ is a toroidal group of order 32 or a quotient thereof. If (i) and (ii) occur, Proulx shows that $K(G, X)$ imbeds in a torus if the subgroup $H=\langle y x, x z\rangle$ has index 2 in $G$. By coset enumeration, $H$ has index at most 2 . Moreover, if we let $u=y x$ and $v=x z$ then $u^{2}=1, v^{4}=1$, and $(u v)^{4}=(y z)^{4}=1$ so that $H$ has genus 0 or 1 by Case Ic for $d=3$. Therefore, even if $H=G$, still $G$ has genus at most 1 .

If two faces of size 8 meet at a vertex either both (i) and (ii) hold or at least one of (iii)-(vi) holds; in any case, $G$ has genus 0 or 1 . If each vertex lies on at most one face of size 8 , then by (*) with $n=9$ and $d=3,3 V / 2+9 \chi<5 F_{4}+F_{8}<$ $5 V / 4+V / 8$. We conclude here that if $V>72|\chi|$ and $F_{6}=0$, then $G$ has genus 0 or 1 .

Finally assume $F_{6} \neq 0$. Of the 6-relators considered in Case IIa above for $d=3$, the only ones that do not immediately imply that $G$ has genus 0 or 1 are $(x z)^{3}$ and $(y z)^{3}$. However, if both of these relators hold, Proulx [6, p. 74] shows $G$ is planar. Therefore, we will assume $(y z)^{3}=1,(x z)^{3} \neq 1$, and $F_{6}<V / 6$. (Recall that we still have the 4-relator $x y x y$.) As before in this case, it is safe to assume $F_{7}=0$. Our previous analysis of the situation when $F_{8} \neq 0$ allows us to assume $F_{8}=0$.

Using the facts that $x$ and $y$ commute and that $y z y=z y z$, Proulx [6, p. 93] reduces the list of 9-relators that do not lead to redundancy to $(x y z)^{3}$. This relator makes $G$ a toroidal group of order 24 . The list of 10-relators is also reduced to the single relator $x y z x z x y z x z$. Here we let $u=x y$. Then $x, z, u$ generate $G$ and $u^{2}=1$, $(x u)^{2}=y^{2}=1$, and $u z x z u z x z=1$; but this is presentation (iv) considered before in this case under $F_{8} \neq 0$ and implies $G$ has genus at most 1 . Finally, Proulx shows that every 11-relator leads either to redundancy or to the relator $(x y z)^{3}$ considered above.

Therefore we assume $F_{n}=0$ for $7 \leqslant n \leqslant 11$ (we still have $(x y)^{2}=1,(y z)^{3}=1$, $\left.F_{4}<V / 4, F_{6} \leqslant V / 6\right)$. By (*) with $n=14$ and $d=3$,

$$
4 V+14 \chi \leqslant 10 F_{4}+8 F_{6}+2 F_{12}+F_{13} \leqslant 10 V / 4+8 V / 6+2 F_{12}+F_{13} \text {. }
$$

Thus if $V>84|\chi|$, then $2 F_{12}+F_{13}>0$. Suppose every face of size 12 or 13 involves at least one and hence by irredundance at least two edges labeled $y$. The total number of edges labeled $y$ available for building faces is $V$. Each 4-relator $(x y)^{2}$ uses 2 edges labeled $y$ and each 6-relator $(x y)^{3}$ uses 3 . Then $2 F_{4}+3 F_{6}+$ $2 F_{12}+2 F_{13}<V$. Combining this inequality with $4 V+14 \chi \leqslant 10 F_{4}+8 F_{6}+2 F_{12}$ $+2 F_{13}$ yields 


$$
4 V+14 \chi-10 F_{4}-8 F_{6} \leqslant 2 F_{12}+2 F_{13} \leqslant V-2 F_{4}-3 F_{6} .
$$

Therefore,

$$
4 V+14 \chi \leqslant V+8 F_{4}+5 F_{6} \leqslant V+2 V+5 V / 6
$$

and so $V \leqslant 84|\chi|$. We conclude that if $V>84|\chi|$ then there is a 12 or 13-relator not involving $y$; therefore $(x z)^{6}=1$. Proulx $\left[6\right.$, p. 92] shows that $(x y)^{2}=(y z)^{3}=$ $(x z)^{6}=1$ gives a toroidal imbedding of $K(G, X)$.

This completes Case IIb and the proof of Theorem 2.

Corollary. If $\gamma(G)>1$, then $|\chi(G)| \geqslant|G| / 84$ and $\gamma(G)>|G| / 168+1$. In particular, the number of groups of a given characteristic $\chi<0$ or given genus $\gamma>1$ is finite.

Proof. Suppose $G$ has characteristic $\chi$ and $X$ is a generating set for $G$ such that $K(G, X)$ imbeds in a surface of Euler characteristic $\chi$. If $X$ is redundant, deleting extra generators from $X$ simply deletes edges from $K(G, X)$ which increases the characteristic of $K(G, X)$ (or at worst, leaves it the same). Therefore, we may assume $X$ is irredundant. By Theorem 2 the order of $G$ is bounded above by $84|x|$. Since $\gamma(G) \geqslant 1-\chi(G) / 2$, the inequality for $\gamma(G)$ follows.

The bound on the characteristic of a group given here is in some sense the best possible. Any group $G$ generated by $x, y, z$ with relators $x^{2}, y^{2}, z^{2},(x y)^{2},(y z)^{3}$, $(x z)^{7}$ has an imbedding in a surface of characteristic $-|G| / 84$. Moreover infinitely many finite groups have such a presentation.

5. Some conjectures. The Schreier coset graph of the subgroup $H$ of the group $G$ with generating set $X$ is defined the same as the Cayley graph, only the vertices of the graph are the right cosets of $H$ rather than the elements of $G$. Gross [3] has shown every graph of even degree is a Schreier coset graph. Thus by Theorem 1 there are an infinite number of Schreier coset graphs having a given genus.

It might appear that Theorem 2 implies there are only finitely many Cayley graphs of a given genus $\gamma>1$. However, the restriction of irredundancy in Theorem 2 allows the possibility of an infinite number of toroidal groups having redundant presentations whose Cayley graphs all have a given genus $\gamma>1$. Also, at some points in the proof of Theorem 2 the given generating set is changed so that one draws conclusions only about the group, not about the given Cayley graph. Nevertheless, the following seems likely to be true.

CONJECTURE 1. There are only finitely many Cayley graphs of a given genus $\gamma>1$ (given characteristic $\chi<0$ ).

Let $C(\gamma)$ (respectively $\bar{C}(\chi)$ ) be the number of groups of genus $\gamma$ (characteristic $\chi)$. By Theorem 2, $C(\gamma)$ and $\bar{C}(\chi)$ are finite for $\gamma>1$ and $\chi<0$. Theorem 2 and refinements thereof should help in the computation of $C(\gamma)$ and $\bar{C}(\chi)$ for small values of $\gamma$ and $\chi$. Proulx has made

Conjecture 2 (Proulx). $C(2)=0$.

It was Proulx's conjecture that led to this paper. There are no groups of genus 2 among the groups of order less than 32. A laborious investigation of the proof of Theorem 2 that we will discuss elsewhere indicates that any group of genus 2 has 
order 36,96 , or 48 . These techniques can probably be more easily used to show $\bar{C}(-1)=0$.

It is known that $C(3) \neq 0$. This leads us to make our final

Conjecture 3. $C(\gamma) \neq 0$ for $\gamma>2$.

\section{REFERENCES}

1. R. P. Baker, Cayley diagrams on the anchor ring, Amer. J. Math. 53 (1931), 645-669.

2. J. L. Gross, Voltage graphs, Discrete Math. 9 (1974), 239-246.

3. __ Every regular graph of even degree is a Schreier coset graph, J. Combinatorial Theory Ser. B 22 (1977), 227-232.

4. H. Levinson and B. Maskit, Special embeddings of Cayley diagrams, J. Combinatorial Theory Ser. B 18 (1975), 12-17.

5. H. Maschke, The representation of finite groups, Amer. J. Math. 18 (1896), 156-194.

6. V. K. Proulx, Classification of the toroidal groups, Ph. D. Dissertation, Columbia University, 1977.

7. Classification of the toroidal groups, J. Graph Theory 2 (1978), 269-273.

8. C. L. Siegel, Topics in complex function theory, Vol. II, Wiley-Interscience, New York, 1971.

9. A. T. White, Graphs, groups, and surfaces, North-Holland, Amsterdam, 1973.

10. __ Graphs of groups on surfaces, in Combinatorial Surveys (Proc. 6th British Combinatorial Conf.), ed. P. J. Cameron, Academic Press, New York, 1977.

Department of Mathematics, Colgate University, Hamilton, New York 13346 